\title{
On complexified mechanics and coquaternions
}

\author{
Dorje C Brody and Eva-Maria Graefe \\ Department of Mathematics, Imperial College London, London SW7 2AZ, UK
}

\begin{abstract}
While real Hamiltonian mechanics and Hermitian quantum mechanics can both be cast in the framework of complex canonical equations, their complex generalisations have hitherto remained tangential. In this paper quaternionic and coquaternionic (split-signature analogue of quaternions) extensions of Hamiltonian mechanics are introduced, and are shown to offer a unifying framework for complexified classical and quantum mechanics. In particular, quantum theories characterised by complex Hamiltonians invariant under space-time reflection are shown to be equivalent to certain coquaternionic extensions of Hermitian quantum theories. One of the interesting consequences is that the space-time dimension of these systems is six, not four, on account of the structures of coquaternionic quantum mechanics.

PACS numbers: 03.65.Aa, 02.30.Fn, 03.65.Ca
\end{abstract}

This paper concerns the relation between complexified classical and non-Hermitian quantum mechanics, and their surprising links to quaternionic and coquaternionic mechanics. The main finding is that complexified mechanical systems with real energies studied extensively in the literature over the past decade can alternatively be thought of as certain coquaternionic extensions of the underlying real mechanical systems. This identification leads to the possibility of employing algebraic techniques of quaternions and coquaternions to tackle some of the challenging open issues in complexified classical and quantum mechanics.

Complex (i.e. non-Hermitian) Hamiltonians have long been employed to describe open quantum systems, decay and scattering phenomena [1]. Further, since the realisation that complex operators respecting space-time reflection (PT) symmetry may possess entirely real spectra [2], there have been considerable research interests in examining both physical and mathematical properties of quantum systems described by non-Hermitian Hamiltonians with real spectra. More recently, the interest in these systems has increased notably, in part owing to experimental realisations of the phenomenon of the PT phase transition and other theoretically predicted effects [3, 4].

Complexified classical mechanics has also been studied intensely both in the context of semiclassical calculations and as a classical analogue of non-Hermitian quantum mechanics [5, 6, 7]. At classical level, complex-extended mechanics is characterised by a phase space spanned by complex canonical variables. The quantum analogue of a phase space - in the strict sense of a symplectic manifold upon which the dynamics is governed by Hamilton's equations of motion - is the space of pure states, i.e. the space 
of rays through the origin of the Hilbert space. On this ray space, the wave equation of Schrödinger in Hermitian quantum mechanics can be expressed as Hamilton's equations of motion [8, 9]. An apparent puzzle in this context is that the analysis of complex extensions of quantum mechanics has thus far been confined to the real state space [10, 11, 12] (with the exception of [13]). Therefore, the nature of complexification considered so far for quantum mechanics has a rather different characteristic from that for classical mechanics. If classical phase spaces can be extended into the complex domain, it seems paradoxical that quantum state space cannot be extended in an analogous manner. (Quaternionic quantum mechanics [14, 15] can be viewed as representing 'complex' extensions of the underlying real mechanics for real (Hermitian) quantum systems, although this is not the point of view commonly adopted.)

In the present paper we propose a new approach to these apparently different complex generalisations by making use of quaternionic and coquaternionic formulations. The key idea is that phase-space variables consist of canonical conjugate pairs; hence a complexified mechanical system necessarily involves pairs of complex numbers. On the other hand, pairs of complex numbers can usefully be treated as a single quaternion. It is natural therefore to enquire whether complexified mechanics can be represented more concisely in terms of quaternions, inasmuch as real phase space variables can more conveniently be represented as a single complex number. A deeper motivation for such a study arises from the fact that symmetry properties of quaternions are closely related to symmetries of (Euclidean) space-time [16]; while complexified dynamical systems discussed here are characterised by Hamiltonians invariant under space-time reflection (PT) symmetry. The appearance of space-time symmetry in both complexified mechanics and quaternionic algebra suggests that the latter might constitute a useful tool for the investigation of the former. A further motivation comes from the observation that if the parity operator is assumed trace free, then the real dimensionality of the Hilbert space of a PT-symmetric quantum system is a multiple of four, not two [17]. This suggests that quaternions a priori are suitable candidates for the characterisation of phase space variables for such theories.

With these observations in mind, we shall explore the possibility that complexified mechanics can alternatively be viewed as a version of quaternionic mechanics. We shall find, perhaps somewhat unexpectedly, that PT-symmetric systems are in fact related to coquaternions, rather than quaternions. In this connection it is worth remarking that symmetries of coquaternions are related to the Lorentz group, rather than Euclidean group, in the sense that every rotation in the Minkowski three-space can be expressed in terms of coquaternions. Hence PT symmetry a priori has more in common with Lorentzian space-time than Euclidean space-time. It follows that PTsymmetric quantum mechanics, although related, are not equivalent to the traditional quaternionic quantum theories of [14, 15].

Our hope is that the approach introduced here may ultimately help to tackle some open issues in the area of complexified mechanics that have only been answered at most partially. These include, among others: The classical-quantum correspondence 
of complexified mechanics [18, 19, 20, 21]; the derivation of statistical mechanics of classical PT-symmetric systems [22]; the characterisation of combined systems (e.g., entanglement) in PT-symmetric quantum mechanics, and the formulation of a theory of space-time that is consistent with complex Hamiltonians.

Quaternions and coquaternions. Although properties of quaternions are well known, we find it convenient to briefly summarise here some of the key relations. The algebra of quaternions involves three imaginary units, denoted here by $i, j, k$, satisfying the relation

$$
i^{2}=j^{2}=k^{2}=i j k=-1,
$$

carved into the stone of the Brougham Bridge by Hamilton in an act of "mathematical vandalism" [23]. It follows that quaternions satisfy the following cyclic relation:

$$
i j=-j i=k, \quad j k=-k j=i, \quad k i=-i k=j .
$$

Hence matrix representations of the three imaginary units are just the Pauli matrices multiplied by the complex number.

A generic quaternion can be represented in the form $q=q_{0}+i q_{1}+j q_{2}+k q_{3}$, where $\left\{q_{i}\right\}_{i=0,1,2,3}$ are all real. It is not difficult to see that quaternions that commute with all other quaternions are reals, and that the totality of quaternions that commute with a given quaternion forms a subset isomorphic to complex numbers. The conjugate of a quaternion $q$ is given by $\bar{q}=q_{0}-i q_{1}-j q_{2}-k q_{3}$. It follows that the squared modulus of $q$ is $\bar{q} q=q_{0}^{2}+q_{1}^{2}+q_{2}^{2}+q_{3}^{2}$, and that the inverse of $q$ is $q^{-1}=\bar{q} /(\bar{q} q)$. A quaternion can be expressed in polar form

$$
q=|q| \mathrm{e}^{\boldsymbol{i}_{q} \theta_{q}}=|q|\left(\cos \theta_{q}+\boldsymbol{i}_{q} \sin \theta_{q}\right),
$$

where $\boldsymbol{i}_{q}=\left(i q_{1}+j q_{2}+k q_{3}\right) /|q|$ and $\theta_{q}=\cos ^{-1}\left(q_{0} /|q|\right)$.

The symmetry properties of quaternions are thus closely related to the group $S U(2)$. The $S U(1,1)$ analogues of quaternions are the coquaternions [24], also known as split quaternions. They satisfy the relation

$$
i^{2}=-1, \quad j^{2}=k^{2}=i j k=+1
$$

in place of (1), and the skew-cyclic relation

$$
i j=-j i=k, \quad j k=-k j=-i, \quad k i=-i k=j
$$

in place of (2). Like quaternions, the conjugate of a coquaternion $q=q_{0}+i q_{1}+j q_{2}+k q_{3}$ is $\bar{q}=q_{0}-i q_{1}-j q_{2}-k q_{3}$. It follows that the squared modulus of a coquaternion is indefinite: $\bar{q} q=q_{0}^{2}+q_{1}^{2}-q_{2}^{2}-q_{3}^{2}$. Unlike quaternions, a coquaternion need not have an inverse $q^{-1}=\bar{q} /(\bar{q} q)$ if it is null, i.e. if $\bar{q} q=0$. The polar decomposition of a coquaternion thus involves trigonometric and hyperbolic functions in the usual way. Unlike quaternions, however, coquaternions do not admit finite-dimensional unitary representations.

Complex formulation of real classical and quantum dynamics. Before we proceed to explore the quaternionic or coquaternionic extensions, it is useful to represent the dynamical theory of elementary mechanical systems in terms of complex phase-space 
variables [25, 26]. We start with a one-dimensional dynamical system, described by real canonical variables $p$ and $x$, and introduce the complex conjugate variables $z=(x+i p) / \sqrt{2}$ and $\bar{z}=(x-i p) / \sqrt{2}$. The canonical equation of motion then reads

$$
i \frac{\mathrm{d} z}{\mathrm{~d} t}=\frac{\partial H}{\partial \bar{z}}
$$

where $H=H(z, \bar{z})$ denotes the Hamiltonian. In this formulation it becomes apparent in which way quantum Schrödinger dynamics can be viewed as a special case of Hamiltonian mechanics [25]. Consider a quantum system characterised more generally by a state vector $|z\rangle$, with components $z_{n}$. The Schrödinger equation, with a Hermitian Hamiltonian $\hat{H}$, can then be written in the form $i \dot{z}_{n}=\mathrm{d} H / \mathrm{d} \bar{z}_{n}$, where $H=\langle z|\hat{H}| z\rangle=$ $\sum_{m n} H_{m n} \bar{z}_{n} z_{m}$ is the expectation value of $\hat{H}$. In particular, the Hamiltonian function of a quantum-mechanical system is bilinear in the canonical variables, that is, it is essentially the Hamiltonian of coupled harmonic oscillators.

With this in mind, we shall devote a great part of the present paper to the investigation of the bilinear Hamiltonian $H=\bar{z} z$, leading to the equation of motion $i \mathrm{~d} z / \mathrm{d} t=z$. The trajectories in the $z$-plane are concentric circles about the origin, traversed with a constant angular velocity, which define a linear critical point called a centre. If we perform a Wick rotation, we obtain $\mathrm{d} z / \mathrm{d} t=z($ or $\mathrm{d} z / \mathrm{d} t=-z)$, which defines a linear critical point called a focus. The trajectories are rays leaving (or approaching) the origin, traversed with radially increasing velocity. More generally, consider the equation $\mathrm{d} z / \mathrm{d} t=b z$, where $b$ is a complex constant. If both the real and imaginary parts of $b$ are nonzero, then the corresponding linear critical point is a vortex, and the orbits consist of concentric spirals leaving (or approaching) the origin traversed with equal radially increasing velocities. Quantum mechanically, all fixed points of unitary dynamics are centers, whereas in a PT-symmetric quantum theory a pair of centres can turn into a pair of foci or vortices upon symmetry breaking.

Complexified classical and quantum mechanics. Let us now summarise briefly the conventional way in which classical and quantum mechanics are extended into the complex domain. The complexified classical mechanics typically starts from the real canonical equations of motion $\dot{p}=-\partial H / \partial x$ and $\dot{x}=\partial H / \partial p$, and considers the complexification $p=p_{0}+i p_{1}$ and $x=x_{0}+i x_{1}$. In terms of the four real variables $\left(p_{0}, x_{0}, p_{1}, x_{1}\right)$ the Hamiltonian now takes the form $H=H_{0}\left(p_{0}, x_{0}, p_{1}, x_{1}\right)+$ $i H_{1}\left(p_{0}, x_{0}, p_{1}, x_{1}\right)$. Assuming that $H$ is analytic and thus satisfies the Cauchy-Riemann conditions $\partial H_{0} / \partial x_{0}=\partial H_{1} / \partial x_{1}, \partial H_{0} / \partial x_{1}=-\partial H_{1} / \partial x_{0}, \partial H_{0} / \partial p_{0}=\partial H_{1} / \partial p_{1}$, and $\partial H_{0} / \partial p_{1}=-\partial H_{1} / \partial p_{0}$, the equations of motion reduce to

$$
\dot{p}_{0}=-\frac{\partial H_{0}}{\partial x_{0}}, \quad \dot{x}_{0}=\frac{\partial H_{0}}{\partial p_{0}}, \quad \dot{p}_{1}=\frac{\partial H_{0}}{\partial x_{1}}, \quad \text { and } \quad \dot{x}_{1}=-\frac{\partial H_{0}}{\partial p_{1}} .
$$

In particular, since the total energy $H_{0}+i H_{1}$ is conserved under the Hamiltonian dynamics, if the initial energy is real so that $H_{1}=0$, then it will remain real. Thus, a complexified Hamiltonian system is equivalent to a real two-dimensional system for which the position and momentum are interchanged in the second dimension [5, 6]. 
The investigations of 'complexified' quantum mechanics, on the other hand, consider complex Hamiltonians of the form $\hat{H}=\hat{H}_{0}+i \hat{H}_{1}$, while typically leaving the quantum phase space real. The equation of motion is still given by the Schrödinger equation. That is, writing $z_{n}=\left(x_{n}+i p_{n}\right) / \sqrt{2}$ for the components of the state vector, we have $i \dot{z}_{n}=\mathrm{d} H / \mathrm{d} \bar{z}_{n}$, where the Hamiltonian function is the expectation value of $\hat{H}$, which is now complex. It is then straightforward to verify that equations of motion for the real phase space variables $p_{n}, x_{n}$ are given by

$$
\dot{p}_{n}=-\frac{\partial H_{0}}{\partial x_{n}}-\frac{\partial H_{1}}{\partial p_{n}} \quad \text { and } \quad \dot{x}_{n}=\frac{\partial H_{0}}{\partial p_{n}}-\frac{\partial H_{1}}{\partial x_{n}} .
$$

This structure is a combination of a Hamiltonian symplectic flow generated by $H_{0}$ and a Hamiltonian gradient flow generated by $H_{1}$, and also appears in the semiclassical limit of non-Hermitian quantum dynamics [18].

The dynamics of complexified quantum systems considered in the literature, for the most part, are characterised by (8), and not by (7). This is the sense in which the nature of complexification in classical mechanics has been different from that of quantum mechanics. To see in which way these two formalisms might be unified by a quaternionic or coquaternionic approach, we shall now investigate the special case of a bilinear Hamiltonian, before turning to the more general case.

Quaternionic and coquaternionic oscillators. We again examine a one-dimensional dynamical system with the Hamiltonian $H=\bar{z} z$, but now $z$ is assumed a quaternion. That is, although the dynamical variable is given by $z=(x+i p) / \sqrt{2}$, we let $p$ and $x$ extend into the complex domain according to the prescription $p \rightarrow p_{0}+j p_{1}$ and $x \rightarrow x_{0}+j x_{1}$. It follows from the algebraic property (2) that $z=\left(x_{0}+i p_{0}+j x_{1}+k p_{1}\right) / \sqrt{2}$. We assume for simplicity a superselection rule [14] that singles out the complex number $i$ for determining the direction of time. Hence the canonical equation of motion is given by (6) with $H=p_{0}^{2}+p_{1}^{2}+x_{0}^{2}+x_{1}^{2}$, and we obtain

$$
\begin{cases}\dot{p}_{0}=-x_{0} & \dot{p}_{1}=-x_{1} \\ \dot{x}_{0}=p_{0} & \dot{x}_{1}=p_{1} .\end{cases}
$$

Thus, a quaternionic oscillator is equivalent to a two-dimensional oscillator. We remark that working with the real variables $(p, x)$, a natural way of extending the theory into quaternionic domain would not have been apparent.

Let us compare this system with a complex PT-symmetric oscillator [7], where we take the Hamiltonian $H=\frac{1}{2}\left(p^{2}+x^{2}\right)$ for which equations of motion read $\dot{p}=-x$ and $\dot{x}=p$, and extend the phase space variables into the complex domain: $p \rightarrow p_{0}+i p_{1}$ and $x \rightarrow x_{0}+i x_{1}$. The resulting dynamical equations are given by (9). While equations of motion agree, and indeed they share the same fixed-point structure, these two theories are nevertheless distinct because of boundary conditions: The energy $E=p_{0}^{2}+p_{1}^{2}+x_{0}^{2}+x_{1}^{2}$ is positive definite for quaternionic oscillators; whereas the energy $E=p_{0}^{2}-p_{1}^{2}+x_{0}^{2}-x_{1}^{2}$ is indefinite for PT-symmetric oscillators.

Next we consider a coquaternionic oscillator, with the same superselection rule. (It is worth remarking that unlike quaternionic quantum mechanics where one has to make 
the choice for the direction of time, in coquaternionic quantum mechanics the imaginary unit $i$ is naturally preferred on account of the fact that $i^{2}=-1$, while $j^{2}=k^{2}=+1$.) In this case, equations of motion remain the same, but owing to the split-signature of coquaternions the energy is given by $E=p_{0}^{2}-p_{1}^{2}+x_{0}^{2}-x_{1}^{2}$. Hence we conclude that a complex PT-symmetric oscillator is equivalent to a coquaternionic oscillator. An important point to note here is the fact that in the complex formulation of mechanics the construction of the Hamiltonian such as $H=\bar{z} z$ implicitly involves the notion of an inner product, which is not evident in Hamilton's formulation involving real variables $(p, x)$. In the case of a complex PT-symmetric oscillator, this inner product is in effect determined by a PT conjugation, which, owing to the fact that the parity operator is trace free, has a split signature equivalent to a coquaternionic norm.

At the level of classical mechanics, the appearance of a PT inner product seems unproblematic because it has no impact on equations of motion. However, if we attempt to formulate statistical mechanics, for instance, then the use of a PT inner product raises a severe obstacle: For any bounded energy, the energy shell in phase space is not compact, hence an equilibrium microcanonical distribution cannot be defined. At the quantum level, the issue of indefiniteness in a PT inner product has been recognised earlier because inner products are related to probabilities, which have to be nonnegative. To remedy this issue, an alternative inner product, based on a CPT conjugation, has been introduced [27, 28]. One possible way forward in the case of a complex classical oscillator therefore is to modify the inner product such that indefinite components pick up an additional minus one. Then energy shells for finite energies become compact, allowing, in particular, for a rigorous formulation of statistical mechanics.

Coquaternionic mechanics with real and complex energies. More generally, consider now a real-valued Hamiltonian $H(z, \bar{z})$ where $z=\left(x_{0}+i p_{0}+j x_{1}+k p_{1}\right) / \sqrt{2}$ is a coquaternion, and hence $\sqrt{2} \partial / \partial \bar{z}=\partial / \partial x_{0}-i \partial / \partial p_{0}+j \partial / \partial x_{1}+k \partial / \partial p_{1}$. From (므) we thus deduce that

$$
i \dot{z}=\left[\frac{\partial H}{\partial x_{0}}+i \frac{\partial H}{\partial p_{0}}-j \frac{\partial H}{\partial x_{1}}-k \frac{\partial H}{\partial p_{1}}\right]
$$

and hence that

$$
\dot{p}_{0}=-\frac{\partial H}{\partial x_{0}}, \quad \dot{x}_{0}=\frac{\partial H}{\partial p_{0}}, \quad \dot{p}_{1}=\frac{\partial H}{\partial x_{1}}, \quad \text { and } \quad \dot{x}_{1}=-\frac{\partial H}{\partial p_{1}} .
$$

Comparing these equations with (7) where the initial energy is assumed real, we find therefore that a PT-symmetric classical system with real energy is equivalent to a coquaternionic mechanical system with real energy. Note that in a quaternionic mechanical system the role of $p_{1}$ and $x_{1}$ are interchanged, and thus it is merely equivalent to a two-dimensional real system.

An alternative case to consider is where the phase space or the state space variables are kept real so that $z$ remains a complex number, but the energy $H=H_{0}+i H_{1}$ is made complex. In this case, it follows from (6) that equations of motion read

$$
\dot{p}=-\frac{\partial H_{0}}{\partial x}+\frac{\partial H_{1}}{\partial p} \quad \text { and } \quad \dot{x}=\frac{\partial H_{0}}{\partial p}+\frac{\partial H_{1}}{\partial x}
$$


which is of course a special case of (8) . If we now allow both energy and phase space variables be complex, then in the coquaternionic case, we obtain:

$$
\begin{aligned}
& \dot{p}_{0}=-\frac{\partial H_{0}}{\partial x_{0}}+\frac{\partial H_{1}}{\partial p_{0}}, \quad \dot{x}_{0}=\frac{\partial H_{0}}{\partial p_{0}}+\frac{\partial H_{1}}{\partial x_{0}}, \\
& \dot{p}_{1}=\frac{\partial H_{0}}{\partial x_{1}}+\frac{\partial H_{1}}{\partial p_{1}}, \quad \dot{x}_{1}=-\frac{\partial H_{0}}{\partial p_{1}}+\frac{\partial H_{1}}{\partial x_{1}} .
\end{aligned}
$$

These equations constitute natural generalisations of (12) in the sense that they embody the structure of a combination of a Hamiltonian symplectic flow generated by $H_{0}$ and a Hamiltonian gradient flow generated by $H_{1}$.

We remark, more generally, that if the Hamiltonian is coquaternionic-valued so that $H=H_{0}+i H_{1}+j H_{2}+k H_{3}$, then (10) implies

$\dot{p}_{0}=-\frac{\partial H_{0}}{\partial x_{0}}+\frac{\partial H_{1}}{\partial p_{0}}+\frac{\partial H_{2}}{\partial x_{1}}+\frac{\partial H_{3}}{\partial p_{1}}, \quad \dot{x}_{0}=\frac{\partial H_{0}}{\partial p_{0}}+\frac{\partial H_{1}}{\partial x_{0}}-\frac{\partial H_{2}}{\partial p_{1}}+\frac{\partial H_{3}}{\partial x_{1}}$,

$\dot{p}_{1}=\frac{\partial H_{0}}{\partial x_{1}}+\frac{\partial H_{1}}{\partial p_{1}}-\frac{\partial H_{2}}{\partial x_{0}}+\frac{\partial H_{3}}{\partial p_{0}}, \quad \dot{x}_{1}=-\frac{\partial H_{0}}{\partial p_{1}}+\frac{\partial H_{1}}{\partial x_{1}}+\frac{\partial H_{2}}{\partial p_{0}}+\frac{\partial H_{3}}{\partial x_{0}}$

for the dynamical equations.

Two oscillators and two-level systems. The orbit space of a classical system of a pair of oscillators is equivalent mathematically (though not physically) to a quantum twolevel system. In particular, the reduced phase space of both systems is just the Bloch sphere $S^{2}$. This can be seen by noting that the Hamiltonian of a pair of uncoupled oscillators in complex coordinates is $H=\bar{z}_{1} z_{1}+\bar{z}_{2} z_{2}$. If the energy is fixed we obtain the three-sphere $\bar{z}_{1} z_{1}+\bar{z}_{2} z_{2}=1$, upon which the Hamiltonian flow acts as scalar multiplication $z_{n} \rightarrow \mathrm{e}^{i t} z_{n}$ [29]. Hence the orbit space is just the two-sphere resulting from the Hopf map $S^{3} \rightarrow S^{2}$.

Similarly, a pair of classical quaternionic oscillators is equivalent to a quaternionic two-level quantum system. In this case, once energy is fixed we obtain the sevensphere $\bar{z}_{1} z_{1}+\bar{z}_{2} z_{2}=1$, upon which the Hamiltonian flow acts as quaternionic scalar multiplication. Hence the associated phase space (Bloch sphere) is a four-sphere $S^{4}$ resulting from the Hopf map $S^{7} \rightarrow S^{4}$. To see the structure of the state space in the quaternionic case more explicitly, it suffices to note that a generic normalised state vector in a quaternionic two-level system can be written in the parametric form

$$
\left(\begin{array}{l}
z_{1} \\
z_{2}
\end{array}\right)=\left(\begin{array}{l}
\cos \frac{1}{2} \theta \\
\sin \frac{1}{2} \theta \mathrm{e}^{i_{\phi} \phi_{1}}
\end{array}\right),
$$

where $\boldsymbol{i}_{\phi}=i \cos \phi_{2}+j \sin \phi_{2} \cos \phi_{3}+k \sin \phi_{2} \sin \phi_{3}$. It is then straightforward to see that $\left(\theta, \phi_{1}, \phi_{2}, \phi_{3}\right)$ constitute spherical coordinates for an $S^{4}$ in $\mathbb{R}^{5}$. In higher dimensions, the state space is just the quaternionic projective Hilbert space endowed with a quaternionic Fubini-Study metric arising from transition probabilities. In the case of a pair of coquaternionic oscillators, the resulting orbit space is hyperbolic (a four-dimensional analogue of the Poincaré disk, which is an example of a Siegel domain [30]).

In the case of a quaternionic or coquaternionic two-level system, a generic Hermitian 
Hamiltonian can be expressed in the form

$$
\hat{H}=\frac{1}{2} \omega \mathbb{1}+\sum_{l=1}^{5} n_{l} \hat{\sigma}_{l},
$$

where $\omega \in \mathbb{R}, \vec{n}$ is a unit vector on $S^{4} \subset \mathbb{R}^{5}$, and

$$
\begin{gathered}
\hat{\sigma}_{1}=\left(\begin{array}{ll}
0 & 1 \\
1 & 0
\end{array}\right), \quad \hat{\sigma}_{2}=\left(\begin{array}{cc}
0 & -i \\
i & 0
\end{array}\right), \quad \hat{\sigma}_{3}=\left(\begin{array}{cc}
1 & 0 \\
0 & -1
\end{array}\right), \\
\hat{\sigma}_{4}=\left(\begin{array}{cc}
0 & -j \\
j & 0
\end{array}\right), \quad \hat{\sigma}_{5}=\left(\begin{array}{cc}
0 & -k \\
k & 0
\end{array}\right)
\end{gathered}
$$

are the (co)quaternionic Pauli matrices. For a quaternionic Hermitian matrix the eigenvalues are real; whereas they are either real or appear as complex conjugate pairs in the case of a coquaternionic Hermitian matrix. In either case, for two-level systems there are six parametric degrees of freedom (consistent with the observation that the most general PT-symmetric $2 \times 2$ Hamiltonian has six exogenous parameters [31]). In standard quantum mechanics, it follows from the construction of spin-orbit interaction (the so-called Pauli correspondence) that one can identify the spin operators with the three spatial directions. Similarly, (17) suggests that in a quaternionic or coquaternionic quantum mechanics, the ambient space has five dimensions.

It is worth remarking that owing to the lack of commutativity of the imaginary units there are "observable" effects in quaternionic or coquaternionic mechanics that have no analogue in real mechanics. For instance, a Hamiltonian proportional to an identity matrix can generate nontrivial dynamics [32]. In particular, if we let $i$ determine the preferred complex structure, and take the Hamiltonian $\hat{H}=\frac{1}{2} \omega \mathbb{1}$, then the dynamics gives rise to a Rabi oscillation between the $\hat{\sigma}_{4}$ and $\hat{\sigma}_{5}$ directions. This can be seen most easily in the Heisenberg picture:

$$
\hat{\sigma}_{4}(t)=\cos (\omega t) \hat{\sigma}_{4}+\sin (\omega t) \hat{\sigma}_{5}
$$

where we have chosen the initial state to be the $\hat{\sigma}_{4}$ eigenstate. Therefore, we can generate dynamics with vanishing energy gap. This is reminiscent of the so-called "arbitrary fast" quantum state transport effect [10, 11, 12].

Let us now consider a $2 \times 2$ coquaternionic Hermitian Hamiltonian and express this in the form

$$
\hat{H}=\left(\begin{array}{cc}
s+t & q \\
\bar{q} & s-t
\end{array}\right),
$$

where $q=q_{0}+i q_{1}+j q_{2}+k q_{3}$. The eigenvalues are $E_{ \pm}=s \pm \sqrt{t^{2}+\bar{q} q}$, where $\bar{q} q=$ $q_{0}^{2}+q_{1}^{2}-q_{2}^{2}-q_{3}^{2}$. Therefore, in the coquaternionic representation, if $t^{2}+q_{0}^{2}+q_{1}^{2}>q_{2}^{2}+q_{3}^{2}$, then the eigenvalues are real, which can be identified as the region of unbroken PT symmetry; otherwise, the eigenvalues form a complex conjugate pair.

Working in the basis for which $\hat{H}$ is diagonal, the phase-space function for the Hamiltonian is $H=\bar{z}_{1} E_{+} z_{1}+\bar{z}_{2} E_{-} z_{2}$ (note that in general $E_{ \pm}$and $z_{i}$ need not commute, 
since $E_{ \pm}$need not be real). It follows that equations of motion are given by $\dot{z}_{1}=i E_{+} z_{1}$ and $\dot{z}_{2}=i E_{-} z_{2}$. In particular, if PT symmetry is unbroken, then eigenvalues are real and the fixed points associated with the dynamical evolution are centres; whereas if the symmetry is broken, then the fixed points form a vortex pair. This is a characteristic feature of the so-called PT phase transition whereby a pair of centres coalesce and turn into a pair of vortices (a sink and a source) [33. Such a transition does not occur in the case of a quaternionic quantum theory, and this establishes the inequivalence of PT-symmetric and quaternionic quantum theories.

Discussion. We have shown that PT-symmetric quantum theories are equivalent to certain coquaternionic extensions of Hermitian quantum theories, at least in finitedimensional cases. This equivalence suggests that the underlying space-time dimension is six, not four, on account of the two additional dimensions generated by the additional imaginary units. This follows from the fact that spin-orbit interaction can only be defined for five spatial dimensions in quaternionic or coquaternionic quantum theories.

Needless to say, there is a substantial literature dealing with various aspects of six-dimensional space-times. Apart from the practicality that calculus on a sixdimensional space facilitates various analysis in four dimensions [34, 35, 36], models for six-dimensional space-times have also been proposed at a fundamental level. Empirical evidences for extra dimensions are often sought at inaccessibly high energies. However, by identifying physical phenomena predicted by coquaternionic quantum theories that cannot be described by standard complex quantum mechanics (such as the Rabi oscillation with vanishing energy gap described above), it is possible to seek compelling evidences for the existence of extra dimensions at low energies (cf. [37] for a discussion on difficulties in detecting quaternionic manifestations in scattering experiments). To this end, it is worth remarking that evidences for octonionic quantum mechanics would suggest that the underlying space-time has dimension ten.

Some of the challenging open issues of PT-symmetric quantum theories include the formulation of combined systems to characterise such notions as entanglement and coherent states. The mathematical difficulty might be rooted in the fact that quaternionic projective Hilbert spaces (unlike complex projective space of ordinary quantum mechanics [38]) do not admit algebraic varieties that characterise subspaces of particular physical characteristics [39]. Nevertheless, progress has been made in the context of quaternionic quantum mechanics to formulate combined systems [40] or coherent states [41, 42. It may be that these techniques can be applied to investigate open issues associated with combined systems for PT-symmetric quantum theories.

EMG is supported by an Imperial College Junior Research Fellowship.

[1] Moiseyev, N. 2011 Non-Hermitian Quantum Mechanics, (Cambridge: Cambridge University Press).

[2] Bender, C. M. \& Boettcher S. 1998 Real spectra in non-Hermitian Hamiltonians having PT symmetry. Phys. Rev. Lett. 80, 5243.

[3] Guo, A. et al. 2009 Observation of PT-symmetry breaking in complex optical potentials. Phys. Rev. Lett. 103, 093902. 
[4] Zhao, K. F., Schaden, M. \& Wu, Z. 2010 Enhanced magnetic resonance signal of spin-polarized $\mathrm{Rb}$ atoms near surfaces of coated cells. Phys. Rev. A81, 042903.

[5] Xavier, A. L. Jr. \& De Aguiar, M. A. M. 1996 Complex trajectories in the quartic oscillator and its semiclassical coherent-state propagator. Ann. Phys. 252, 458.

[6] Kaushal, R. S. \& Korsch, H. J. 2000 Some remarks on complex Hamiltonian systems. Phys. Lett. A 276, 47.

[7] Bender, C. M., Boettcher S. \& Meisinger, P. N. 1999 PT-symmetric quantum mechanics. J. Math. Phys. 40, 2201.

[8] Strocchi, F. 1966 Complex coordinates and quantum mechanics. Rev. Mod. Phys. 38, 36.

[9] Weinberg, S. 1989 Testing quantum mechanics. Ann. Phys. 194, 336.

[10] Bender, C. M., Brody, D. C., Jones, H. F. \& Meister, B. K. 2007 Faster than Hermitian quantum mechanics. Phys. Rev. Lett. 98, 040403.

[11] Mostafazadeh, A. 2007 Quantum Brachistochrone problem and the geometry of the state space in pseudo-Hermitian quantum mechanics. Phys. Rev. Lett. 99, 130502.

[12] Günther, U. \& Samsonov, B. F. 2008 Naimark-dilated PT-symmetric Brachistochrone. Phys. Rev. Lett. 101, 230404.

[13] Nesterov, A. I. 2009 Non-Hermitian quantum systems and time-optimal quantum evolution. SIGMA 5, 069.

[14] Finkelstein, D., Jaueh, J. M., Schiminovieh, S. \& Speiser, D. 1962 Foundations of quaternion quantum mechanics. J. Math. Phys. 3, 207.

[15] Adler, S. L. 1995 Quaternionic Quantum Mechanics and Quantum Fields. (Oxford: Oxford University Press).

[16] Conway, J. H. \& Smith, D. A. 2003 On Quaternions and Octonions. (Wellesley, MA: A K Peters).

[17] Bender, C. M., Brody, D. C., Hughston, L. P. \& Meister, B. K. 2007 Geometry of PT-symmetric quantum mechanics. arXiv:0704.2959

[18] Graefe, E. M., Höning, M. \& Korsch, H. J. 2010 Classical limit of non-Hermitian quantum dynamics - a generalized canonical structure. J. Phys. A43, 075306.

[19] Bender, C. M., Hook, D. W., Meisinger, P. N. \& Wang, Q. 2010 Complex correspondence principle. Phys. Rev. Lett. 104, 061601.

[20] Mostafazadeh, A. 2006 Real description of classical Hamiltonian dynamics generated by a complex potential. Phys. Lett. A357, 177.

[21] Curtright, T. \& Mezincescu, L. 2007 Biorthogonal quantum systems. J. Math. Phys. 48, 092106.

[22] Jones, H. F. \& Moreira, E. S. 2010 Quantum and classical statistical mechanics of a class of non-Hermitian Hamiltonians. J. Phys. A43, 055307.

[23] Baez, J. 2002 The octonions. Bull. Amer. Math. Soc. 39, 145.

[24] Cockle, J. 1849 On systems of algebra involving more than one imaginary. Phil. Magazine 35, 434.

[25] Mackey, G. W. 1963 Mathematical Foundations of Quantum Mechanics (New York: W.A. Benjamin).

[26] Riccia, G. D. \& Wiener, N. 1966 Wave mechanics in classical phase space, Brownian motion, and quantum theory. J. Math. Phys. 71372.

[27] Bender, C. M., Brody, D. C. \& Jones, H. F. 2002 Complex extension of quantum mechanics. Phys. Rev. Lett. 89, 270401.

[28] Mostafazadeh, A. 2002 Pseudo-Hermiticity versus PT-symmetry III: Equivalence of pseudoHermiticity and the presence of antilinear symmetries. J. Math. Phys. 43, 3944.

[29] Atiyah, M. F. 1983 Angular momentum, convex polyhedra, and algebraic geometry. Proc. Edinburgh Math. Soc. 26, 121.

[30] Vinberg, É. B., Gindikin, S. G. and Pjateckii-S̆apiro, I. I. 1963 Classification and canonical realisation of complex bounded homogeneous domains. Trudy Moskov. Mat. Obšc 12, 359.

[31] Wang, Q., Chia, S., \& Zhang, J. 2010 PT symmetry as a generalisation of Hermiticity. J. Phys. A43, 295301. 
[32] Walff, U. 1981 A quaternion quantum system. Phys. Lett. A84, 89.

[33] Graefe, E. M., Korsch, H. J. \& Niederle, A. E. 2010 Quantum-classical correspondence for a non-Hermitian Bose-Hubbard dimer. Phys. Rev. A 82, 013629.

[34] Dirac, P. A. M. 1936 Wave equations in conformal space. Ann. Math. 37, 429.

[35] Hughston, L. P. \& Hurd, T. R. 1983 A CP ${ }^{5}$ calculus for space-time fields. Phys. Rep. 100273.

[36] Weinberg, S. 2010 Six-dimensional methods for four-dimensional conformal field theories. Phys. Rev. D82 045031.

[37] Adler, S. L. 1988 Scattering and decay theory for quaternionic quantum mechanics, and the structure of induced $T$ nonconservation. Phys. Rev. D37, 3654.

[38] Brody, D. C. \& Graefe, E. M. 2010 Coherent states and rational surfaces. J. Phys. A43, 255205.

[39] Berndt, J. 2003 Symmetric submanifolds of symmetric spaces. Proc. Seventh International Workshop on Diff. Geom. 7, 1.

[40] Razon, A. \& Horwitz, L. P. 1991 Tensor product of quaternion Hilbert modules. Act. App. Math. 24, 141.

[41] Adler, S. L. \& Millard, A. C. 1997 Coherent states in quaternionic quantum mechanics. J. Math. Phys. 38, 2117.

[42] Thirulogasanthar, K., Honnouvo, G. \& Krzyzak, A. 2010 Coherent states and Hermite polynomials on quaternionic Hilbert spaces. J. Phys. A43, 385205. 